\title{
PERFIL DOS PACIENTES INTERNADOS NA UNIDADE DE TERAPIA INTENSIVA NEONATAL
}

\author{
PROFILE OF PATIENTS ADMITTED TO THE NEONATAL INTENSIVE CARE UNIT
}

Ana Cristina Zulian ${ }^{1}$, Débora $D^{\prime}$ Agostini Jorge Lisboa ${ }^{2}$, Juliana Schecci ${ }^{3}$, Renato Ravizon Lisboa ${ }^{4}$

${ }^{1}$ Acadêmica de Fisioterapia pela Universidade de Passo Fundo, RS. Brasil.

${ }^{2}$ Fisioterapeuta. Mestre em Envelhecimento Humano pela Universidade de Passo Fundo. Docente da Universidade de Passo Fundo- RS. Passo Fundo, RS, Brasil. debydj@yahoo.com.br

${ }^{3}$ Mestre em Envelhecimento Humano pela Universidade de Passo Fundo. Docente do curso de Fisioterapia da Universidade de Passo Fundo- RS. Passo Fundo, RS, Brasil. Juliana.schecci@upf.br

${ }^{4}$ Médico. Especialista em Clínica médica e Medicina da família e comunidade. Prefeitura Municipal de Passo Fundo. rrlisboa@yahoo.com.br

\section{ABSTRACT}

The elaboration of the epidemiological profile is an important tool to recognize the needs of a population, making it possible to plan actions for prevention, control or even disease eradication. OBJETIVE: to trace the profile of patients admitted to the Neonatal Intensive Care Unit (NICU) of Hospital de Clinicas de Passo Fundo, in the northern state of Rio Grande do Sul. METHODOLOGY: The research is descriptive, prospective, sectional and quantitative. Data were collected from the medical records of all patients admitted to the NICU from August to October 2016. RESULTS: The sample consisted of 32 individuals, predominantly female, with a higher incidence of cesarean delivery and the main cause of hospitalization was due to the prematurity associated with hyaline membrane disease. The lowest gestational age corresponded to 26 weeks and the highest to 40 weeks, and in relation to the maternal age, the minimum was 16 years and the maximum of 37 years. Prenatal followup was performed by 30 pregnant women with an average number of visits of 7.3. From the 32 hospitalized individuals, 21 required mechanical ventilation, with prevalence for females and the mean time of use of 7.2 days. Only 13 neonates were prescribed physiotherapy. CONCLUSIONS: The epidemiological profile of patients admitted to the Neonatal Intensive Care Unit of a large hospital in a city in the interior of the state of Rio Grande do Sul was characterized by the predominance of female newborns with a gestational age of 34 weeks, with prematurity associated with Membrane Disease being the main diagnosis. The final outcome was predominance of discharge to the ward.Keywords: Intensive care units. Infant, newborn. Infant, Premature. Epidemiological profile. 


\section{RESUMO}

A elaboração do perfil epidemiológico é uma ferramenta importante para o reconhecimento das necessidades de uma população, tornando possível planejar ações para prevenção, controle ou até erradicação de doenças. OBJETIVO: traçar o perfil dos pacientes internados na Unidade de Terapia Intensiva Neonatal (UTIN) do Hospital de Clínicas de Passo Fundo, no norte do estado do Rio Grande do Sul. METODOLOGIA: pesquisa descritiva, prospectiva, transversal e quantitativa. Os dados foram coletados dos prontuários clínicos de todos os pacientes que internaram na UTIN, no período de agosto a outubro de 2016. RESULTADOS: a amostra foi composta de 32 indivíduos, tendo predominância do sexo feminino, maior ocorrência de parto cesáreo e a principal causa de internação foi devido à prematuridade associada à doença da membrana hialina. A menor idade gestacional correspondeu a 26 semanas e a maior 40 semanas. Em relação à idade materna, a mínima foi 16 anos e a máxima de 37 anos. $O$ acompanhamento pré-natal foi realizado em 30 gestantes, com um número médio de consultas de 7,3. Dos 32 indivíduos internados, 21 necessitaram de ventilação mecânica, com predomínio do sexo feminino e tempo médio de uso de 7,2 dias. Apenas 13 neonatos tiveram prescrição de fisioterapia. CONCLUSÃO: o perfil epidemiológico dos pacientes internados na Unidade de Terapia Intensiva Neonatal de um hospital de grande porte de uma cidade do interior do estado do Rio Grande do Sul foi caracterizado por predomínio de neonatos do sexo feminino, com idade gestacional de 34 semanas, tendo prematuridade associada à Doença da Membrana como principal diagnóstico, e desfecho final de alta para enfermaria.

Palavras-chave: Unidade de terapia intensiva. Recém-nascido. Prematuridade. Perfil epidemiológico.

\section{Como citar este artigo:}

Zulian AC, Lisboa DDAJ, Schecci J, Lisboa RR. Perfil dos pacientes internados na Unidade de Terapia Intensiva Neonatal. J. Ciênc. Saúde [internet]. 2018 [acesso em: dia mês abreviado ano];1(3):38-48. Disponível em: DOI: https://doi.org/10.26694/2595-0290.20181338-488315

\section{(cc)BY}




\section{INTRODUÇÃO}

Os cuidados destinados ao recém-nascido (RN) imediatamente após o nascimento são primordiais para a adaptação do bebê ao novo meio, diminuindo a morbi-mortalidade neonatal. O momento da transição do meio intra para o extrauterino é caracterizado por diversas alterações para o $\mathrm{RN}$, tornando-o frágil e debilitado. O meio intrauterino proporciona um ambiente seguro, aconchegante, com temperatura e luminosidade apropriadas, com ruídos suaves, não exigindo esforços para executar as funções vitais. Após o parto, o bebê vai se habituando progressivamente ao meio extrauterino ultrapassando as dificuldades ligadas ao seu desenvolvimento ${ }^{(1)}$.

Quando o bebê necessita sair precocemente do útero da mãe, as alterações fisiológicas normais precisam sofrer adaptações antes do período esperado, acarretando ao recém-nascido amadurecimento precoce e possíveis complicações ao longo da vida. As crianças que sobrevivem a essas condições adversas de nascimento possuem uma elevada morbimortalidade a longo prazo, incluindo atraso no desenvolvimento neuropsicomotor, quando comparadas com crianças nascidas no período considerado a termo, após 37 semanas $^{(2)}$.

Quando o bebê necessita sair precocemente do útero da mãe, as alterações fisiológicas normais precisam sofrer adaptações antes do período esperado, acarretando ao recém-nascido amadurecimento precoce e possíveis complicações

Segundo Rodrigue e Belham (2017), a Unidade de Terapia Intensiva Neonatal (UTIN) é um setor de assistência aos Recém-nascidos (RNs) graves ou potencialmente graves, que estão vulneráveis e precisam de cuidados específicos e contínuos, sendo esta uma unidade dotada de condições técnicas adequadas à prestação de assistência especializada, incluindo instalações físicas, equipamentos e recursos humanos. Para o mesmo autor, o desenvolvimento de novas tecnologias proporciona maiores ganhos para os $\mathrm{RNs}$, disponibilizando maiores cuidados e aumento na sobrevida desses indivíduos. Acredita-se que múltiplos fatores impulsionaram o aumento da incidência de partos prematuros, incluindo aumento da idade média materna, uso de métodos de reprodução assistida e maior número de indicações de cesarianas por doenças maternas ou fetais ${ }^{(2)}$.

É sabido que a mortalidade infantil é o principal problema relacionado a prematuridade. Embora tenham ocorrido diversos progressos em relação aos diagnósticos pré-natais, o baixo peso ao nascer (BPN) e a prematuridade perduram como fatores centrais de morbidade e mortalidade no primeiro ano de vida. Tais riscos têm se evidenciado mais severamente nas jovens com idade inferior a 20 anos de idade, particularmente naquelas com menos de 15 anos ou naquelas com idade mais avançada, acima de 35 anos $^{(3,4)}$.

A UTIN é um ambiente destinado para tratamento de bebês prematuros e crianças que apresentam algum transtorno ao nascer. É um local do hospital individualizado, que possui infraestrutura e equipe multiprofissional especializada e qualificada para atender essa população, devido à gravidade do estado de saúde do paciente e a imprevisibilidade ${ }^{(2,5)}$.

Reis, Cavalcante e Santos (2018), observaram em seu estudo que o momento do parto e o primeiro mês de vida são cruciais para o desenvolvimento neonatal, necessitando de tratamento e conduta distintos dentro da UTIN. Muitas vezes, o fato do bebê estar internado na UTIN não significa que o mesmo está adoecido. Em diversos casos, o RN está apenas desenvolvendo suas funções e se capacitando a realizá-las sozinho, como por exemplo, respirar, sugar e deglutir ${ }^{(5)}$.

Neste contexto, devido a importância do tema abordado e da necessidade da realização de uma abordagem multiprofissional, o presente estudo tem como objetivo identificar o perfil dos pacientes internados na UTIN do Hospital de Clínicas de Passo Fundo/RS. 


\section{METODOLOGIA}

Estudo descritivo, transversal e quantitativo, realizado na UTIN de um hospital de grande porte, possuindo 230 leitos, localizado na região norte do estado do Rio Grande do Sul, Brasil. Consiste em um hospital de ensino, com serviços de residência médica e residência multiprofissional em saúde nas áreas de fisioterapia, enfermagem, psicologia e farmácia. É um hospital com serviço de alta complexidade em neurologia, oncologia e cardiologia, servindo de referência para municípios da região sul do Brasil. Possui, ainda, serviço especializado de terapia intensiva, dispondo de 18 leitos na unidade adulta, sendo cinco leitos (27\%) disponibilizados para pacientes cardiológicos cirúrgicos e clínicos, e dez leitos na unidade neonatal. A unidade de Terapia Intensiva Neonatal consiste em uma unidade de terapia intensa (UTI) geral destinando-se ao atendimento de diferentes patologias neonatais.

A pesquisa foi realizada de agosto a outubro de 2016 . Foram incluídos no estudo todos os prontuários dos recém-nascidos que internaram no período da pesquisa. Os dados foram coletados através de análise do prontuário médico dos pacientes, com coleta dos dados por ficha elaborada pelos próprios pesquisadores, em que foram analisados os seguintes dados: sexo, tipo de parto, patologia, desfecho final, idade gestacional, idade materna, realização de prénatal, APGAR do primeiro e quinto minuto, necessidade de ventilação mecânica, tipo de fisioterapia prescrita e frequência, data, motivo da admissão e alta hospitalar.

Após a coleta, os dados foram codificados e digitados pelos pesquisadores em planilha do MS Excel. As variáveis categóricas foram descritas como frequência absoluta e relativa. As variáveis numéricas foram descritas como média \pm desvio padrão ou mediana (percentil25 - percentil75), conforme distribuição normal ou não normal.

A pesquisa foi aprovada pelo comitê de ética e pesquisa da Universidade de Passo Fundo sob protocolo 1.648.989, seguiu as diretrizes $466 / 12$ do Conselho Nacional de Saúde de 2012.

\section{RESULTADOS}

Fizeram parte da amostra 32 indivíduos que internaram na UTIN entre o período de agosto a outubro de 2016, sendo a maioria do sexo feminino (68,75\%). Entre os nascidos de parto cesariana, $69,5 \%$ eram do sexo feminino, entre os que nasceram de parto normal $66,7 \%$ eram do sexo feminino.

Em relação ao perfil das parturientes, $30(93,7 \%)$ realizaram pré-natal, com 4 mulheres das que realizaram pré-natal não sabendo informar quantas consultas havia realizado. O número médio de consultas foi de $7,3( \pm 4,40)$ por mulher, entre as que realizaram o pré-natal e tinham conhecimento do número de consultas realizadas. Em relação a idade materna, observou-se que a menor idade foi de 16 anos, sendo que a menor idade gestacional encontrada foi de 26 semanas (Tabela 1).

A causa mais frequente de internação na UTIN foi prematuridade (PMT) associada à doença da Membrana Hialina (DMH), correspondendo a $18(56,3 \%)$ pacientes, onde $5(15,7 \%)$ eram do sexo masculino e $13(40,6 \%)$ do sexo feminino. Outros motivos menos frequentes foram: PMT associada à RN pequeno para idade.

Tabela 1 - Caracterização gestacional - Passo Fundo, RS 2016.

\begin{tabular}{lccccc}
\hline & Média & Mediana & Desvio Padrão & Mínimo & Máximo \\
\hline Idade materna (anos) & 28,1 & 29,0 & 5,41 & 16 & 37 \\
\hline Número de consultas & 7,3 & 6,0 & 4,40 & 3 & 23 \\
\hline $\begin{array}{l}\text { Idade gestacional } \\
\text { (semanas) }\end{array}$ & 34,0 & 35,0 & 3,12 & 26 & 40 \\
\hline
\end{tabular}


Fonte: elaborado pelo autor 
A Tabela 2 apresenta os dados em relação ao APGAR do primeiro e do quinto minuto após o nascimento, sendo que a maioria dos indivíduos de ambos os sexos obtiveram valores considerados ótimos nos dois tempos avaliados.

Tabela 2 - Valores do APGAR distribuídos por sexo - Passo Fundo, RS 2016.

\begin{tabular}{|c|c|c|c|c|c|c|c|c|}
\hline & \multicolumn{4}{|c|}{ Masculino } & \multicolumn{4}{|c|}{ Feminino } \\
\hline & \multicolumn{2}{|c|}{19 minuto } & \multicolumn{2}{|c|}{ 5o minuto } & \multicolumn{2}{|c|}{10 minuto } & \multicolumn{2}{|c|}{ 50 minuto } \\
\hline & $n$ & $\%$ & $\mathrm{n}$ & $\%$ & $n$ & $\%$ & $\mathbf{N}$ & $\%$ \\
\hline 0 a $3^{*}$ & 1 & 10 & 0 & 0 & 3 & 13,7 & 0 & 0 \\
\hline $4 a^{* *}$ & 3 & 30 & 1 & 10 & 8 & 36,3 & 4 & 18,2 \\
\hline $7^{* * *}$ & 0 & 0 & 2 & 20 & 3 & 13,7 & 0 & 0 \\
\hline 8 a $10^{* * * *}$ & 6 & 60 & 7 & 70 & 8 & 36,3 & 18 & 81,8 \\
\hline
\end{tabular}

Fonte: elaborado pelo autor.

"Dificuldade grave; ${ }^{* *}$ Dificuldade moderada; ${ }^{* * *}$ Dificuldade leve; ${ }^{* * * *}$ Ótimas condições

A causa mais frequente de internação na UTIN foi prematuridade (PMT) associada à doença da Membrana Hialina (DMH), correspondendo a $18(56,3 \%)$ pacientes, onde $5(15,7 \%)$ eram do sexo masculino e $13(40,6 \%)$ do sexo feminino. Outros motivos menos frequentes foram: PMT associada à RN pequeno para idade gestacional (PIG); atresia de esôfago; sepse associada à pneumonia aspirativa; obstrução intestinal; PMT associada à parada cardiorrespiratória (PCR); anóxia neonatal e sepse; hérnia diafragmática; infecção neonatal e Síndrome de Down; Taquipnéia transitória; PMT associada à lábio leporino e fenda palatina completa.
Dos 32 indivíduos, 21 (65,6\%) estavam em ventilação mecânica (VM), 16 (76,1\%) do sexo feminino, sendo o tempo médio de permanência em ventilação de 7,2 $( \pm 11,2)$ dias, com tempo mínimo de 1 dia e máximo de 43 dias.

Apenas $13(40,6)$ indivíduos tiveram prescrição de fisioterapia respiratória, em que 3 (23\%) indivíduos realizaram duas vezes por dia e $10(77 \%)$ realizaram três vezes por dia.

Em relação ao desfecho final (Tabela 3), a maioria dos neonatos foi transferida para os leitos, seguido de permanência das internações na UTIN, alta e óbito, respectivamente. 
Tabela 3 - Desfecho final - Passo Fundo, RS 2016.

\begin{tabular}{lllcc}
\hline & \multicolumn{2}{c}{ Masculino } & \multicolumn{2}{c}{ Feminino } \\
\hline & $\mathbf{n}$ & $\%$ & $\mathbf{n}$ & $\%$ \\
\hline $\begin{array}{l}\text { Transferência para } \\
\text { Leitos de Internações }\end{array}$ & 6 & 60 & 15 & 68,2 \\
\hline Alta direto para casa & 1 & 10 & 0 & 0 \\
\hline Óbito & 0 & 0 & 2 & 9,1 \\
\hline Permanência na UTIN & 3 & 30 & 5 & 22,7 \\
\hline TOTAL INCLUIR & 10 & 100 & 22 & 100 \\
\hline
\end{tabular}

Fonte: elaborado pelo autor.

\section{DSICUSÃO}

No estudo realizado por Rodrigue e Belham (2017)(2), foram admitidos 343 recém-nascidos, sendo o sexo masculino o predominante, totalizando 192 (56\%) internações. Basso, Neves e Silveira $(2012)^{(4)}$ em seu estudo, também constataram predomínio de recémnascidos do sexo masculino, com $52 \%$ internados na UTIN. Esses resultados são diferentes daqueles encontrados nesta pesquisa, em que o sexo feminino foi mais frequente. Entretanto, de modo semelhante ao encontrado neste estudo, Reis, Cavalcante e Santos $(2018)^{(5)}$ observaram predomínio do sexo feminino 13 (65\%) em um grupo de 20 pacientes admitidos na UTIN de um hospital de Cuiabá.

Em nosso estudo, observamos como causa mais frequente de internação na UTIN a prematuridade associada a DMH, corroborando o estudo de Rodrigue e Belham $(2017)^{(2)}$, onde a causa mais frequente de internação foi a prematuridade $(72,7 \%)$, e o Marques et al. $(2018)^{(6)}$, os quais encontraram como principal motivo de admissão a prematuridade, com mais de metade dos neonatos apresentaram algum tipo de infecção durante o período de hospitalização. Já no estudo de Basso, Neves e Silveira (2012)(4) os principais motivos de internação foram as causas respiratórias, com $43,1 \%$, seguidas pelo baixo peso ao nascer, perfazendo $19,3 \%$, e apenas $12,9 \%$ pela prematuridade.

O Brasil apresenta as mais altas taxas de cesarianas no mundo. O parto cesáreo teve muitos avanços nos últimos anos, tornando-se uma prática segura que atualmente conta com poucos riscos, mas não o isenta. A Organização Mundial de Saúde (OMS) alerta que o excesso de cesarianas aumenta o índice de mortalidade de mães e de crianças no país ${ }^{(7,8)}$. Existem indicações de cesariana com o objetivo de aumentar a segurança materno fetal em algumas situações de parto. Algumas vezes este procedimento é indicado de acordo com as condições maternas, outras, pelas condições fetais ${ }^{(9)}$.

Iniciar o pré-natal precocemente é relevante para que a gestante seja estimulada a comparecer às consultas e realizar os exames complementares solicitados. Em termos quantitativos, para um pré-natal com mínimos requisitos de adequação, é recomendada pelo Ministério da Saúde a realização de, pelo menos, seis consultas $^{(10)}$.

Segundo Tase ${ }^{(11)}$, iniciar tardiamente o pré-natal pode estar associado a dificuldades e complicações tanto para mãe como para o bebê, que vão desde a falta de preparação dos serviços até inexperiência da equipe. Para Victora e Cesar ${ }^{(12)}$, um pré-natal efetivo necessita 
de serviços de saúde acessíveis no sentido de localização geográfica e horários de funcionamento, contando com baixo custo e qualidade na assistência dos profissionais, promovendo a redução da morbimortalidade materno-infantil.

Para Romero, Simão e Souza ${ }^{(13)}$, a idade da mãe pode desempenhar influência tanto para as condições do nascimento da criança quanto na saúde da própria puérpera. Gestantes com idade inferior a 17 anos e superior a 35 anos constituem-se em grupo de risco considerável na gravidez ${ }^{(14)}$. Marques et al $(2018)^{(6)}$ observaram em seu estudo quanto aos fatores maternos, uma idade mínima de 15 anos, com idade máxima de 42 anos, e média de 29 anos, corroborando resultados encontrados no presente estudo. Os malefícios de uma gestação precoce ou tardia são definidos, mais vigorosamente, por fatores psicossociais do que biológicos e obstétricos propriamente ditos ${ }^{(15)}$.

Segundo Delpisheh et al. ${ }^{(16)}$, mulheres com idade acima de 35 anos, comparadas com mulheres jovens, apresentam mais abortamentos espontâneos e induzidos, elevado risco de mortalidade perinatal, baixa vitalidade do recém-nascido, baixo peso ao nascer, parto prematuros e fetos pequenos para idade gestacional, resultados estes encontrados na atual pesquisa.

Cabe ressaltar que, nesta pesquisa, a média de idade gestacional foi de 34 semanas, evidenciando-se a ocorrência de um grande número de partos pré-termo. Estudos demonstram que os recém-nascidos pré-termo extremos, podem ter atraso em seu desenvolvimento neuromotor e cognitivo, devido às suas características biológicas relacionadas a idade gestacional e peso ao nascer, ou necessitar mais intervenções e apresentar mais complicações no período neonatal ${ }^{(17,18)}$.

O Índice de APGAR, a idade gestacional e o peso ao nascer estão diretamente relacionados com a sobrevida e, combinados, são um parâmetro de bem-estar do neonato, da qualidade da reanimação, do tamanho e da maturidade do recém-nascido ${ }^{(19)}$.

O Escore de APGAR, ou Índice de APGAR, é utilizado na avaliação imediata do recém-nascido e executado logo após o parto, geralmente no primeiro e no quinto minuto de vida(20), avaliando o estado fisiológico e a capacidade de resposta do $\mathrm{RN}$, auxiliando na identificação da necessidade de reanimação ou algum outro procedimento especial(20,21), podendo, ainda, auxiliar no diagnóstico de asfixia neonatal ${ }^{(21)}$. Recémnascidos com APGAR baixo são aqueles que necessitam de cuidados adicionais, suporte ventilatório ou via aérea artificial. Índice de APGAR inferior a 7 é um sinal de perigo conforme a alteração fisiopatológica e da maturidade do bebê $\hat{e}^{(22,23)}$. Quando este índice estiver for superior a 7 mostra que a criança é sadia e que, provavelmente, não desenvolverá problemas futuros $^{(23)}$.

No estudo realizado por Muniz et al. $(2018)^{(22)}$, com relação ao índice de APGAR, foi observado que 69,1\% $(n=123)$ dos pacientes tinham índice menor ou igual a sete; $22,47 \%(n=40)$, tinham escore superior a sete e $8,43 \%$ ( $n=15$ ) não tinham registro de APGAR no primeiro minuto. Visto que há uma estreita relação com alterações indesejáveis para o RN, sugere-se que o valor de APGAR igual ou menor que sete, no estudo em questão, tem uma relação para a debilitação do quadro de saúde do bebê e, consequentemente, os possíveis óbitos, dados divergentes dos encontrados no presente estudo, sendo que a maioria dos indivíduos de ambos os sexos obtiveram valores considerados ótimos no primeiro e quinto minuto.

Prematuros com idade gestacional inferior a 28 semanas também sofrem maior risco de serem acometidos pela doença da membrana hialina (DMH). A $\mathrm{DMH}$ dá-se por um comprometimento pulmonar secundário a insultos físicos e bioquímicos, com alteração da função do surfactante e formação de membranas hialinas no interior dos alvéolos ${ }^{(24)}$. No presente estudo, devido a média da idade gestacional ser 34 semanas, observou-se um alto índice de prematuridade associado a $\mathrm{DMH}$. A Sociedade Brasileira de Pediatria ${ }^{(24)}$ relata que, quanto mais prematuro for o neonato, maior a gravidade e a possibilidade de desenvolver a DMH.

Basso, Neves e Silveira (2012) $)^{(4)}$ observaram em seu estudo que o tempo de internação dos neonatos variou de um a 160 dias, sendo que $57 \%$ permaneceram na 
unidade entre um e 15 dias, enquanto que $36 \%$ estiveram internados entre 16 e 50 dias. Ressalta-se que apenas $1 \%$ permaneceu por mais de 100 dias na unidade. Nesse sentido, a média do tempo de internação dos neonatos na UTIN foi de 19,6 dias. Entre os sobreviventes, a média de tempo de internação foi de 20,1 dias, e os que foram a óbito tiveram a média de 16,7 dias.

O uso de ventilação mecânica no período neonatal pode causar alterações das vias respiratórias, principalmente através de barotrauma, volutrauma, atelectotrauma e biotrauma. Essas lesões produzidas num período precoce da vida podem ser irreversíveis, mesmo após a maturação pulmonar e o remodelamento das vias aéreas em etapas posteriores ${ }^{(25)}$. Vrijlandt et al. ${ }^{(26)}$, estudaram, na Holanda, 690 crianças que nasceram prematuras no ano de 1983 . Essas crianças foram reavaliadas posteriormente, no ano de 2002 . Os autores concluíram que as crianças nascidas com idade gestacional inferior a 32 semanas e que usaram suporte ventilatório por tempo superior a 28 dias apresentaram cinco vezes mais chance de desenvolver dispneia ao longo da vida, do que aquelas que usaram suporte ventilatório por tempo inferior a 28 dias.

Na prática fisioterapêutica é habitual o contato com limitações, alterações e sequelas dos pacientes, exigindo assim, um complexo nível de conhecimento e experiência técnico-científica(27). Há alguns anos, a fisioterapia respiratória vem sendo executada nas Unidades de Terapia Intensiva Neonatal com o propósito de auxiliar os recém-nascidos em sua recuperação e prevenir eventuais agravos e complicações respiratórias. A fisioterapia respiratória na UTIN tem como objetivo evitar a obstrução brônquica, a hiperinsuflação pulmonar, prevenir alterações na ventilação/perfusão, evitando o aumento do trabalho respiratório, intubação e reintubação(28). Várias técnicas fisioterápicas podem ser utilizadas com os neonatos, como drenagem postural, técnicas de higiene brônquica, reexpansão pulmonar e posicionamento, todas apresentando vantagens e desvantagens ${ }^{(29)}$. Em virtude da falta de maiores estudos na área neonatal, ainda não existe um consenso sobre as técnicas mais indicadas para essa população.
Em relação aos desfechos, Rodrigue e Belham (2017) ${ }^{(2)}$, observaram que 69 (20\%) pacientes evoluíram para óbito durante a internação, tendo 49 destes ocorrendo nas primeiras 72 horas de internação. No estudo de Basso, Neves e Silveira $(2012)^{(4)}$, dos 346 recémnascidos internados na UTIN no período do estudo, 49 foram a óbito, representando $14,2 \%$ da população, indo de encontro ao presente estudo, onde foi observado apenas 2 óbitos no período da pesquisa.

No estudo de Tiago, Caldeira e Vieira ${ }^{(30)}$, mostra-se que para assegurar a sobrevida desses bebês é preciso um longo período de atenções e cuidados intra e extrahospitalares, com o uso de técnicas e procedimentos adequados e capazes de proporcionar mais qualidade de vida a estes bebês prematuros, auxiliando na recuperação da condição dos neonatos internados, prevenindo complicações oriundas de tratamento intensivo e, sobretudo, do longo tempo de internação.

\section{CONCLUSÃO}

Com a realização deste estudo, pode-se elaborar o perfil epidemiológico dos pacientes internados na Unidade de Terapia Intensiva Neonatal de um hospital de grande porte de uma cidade do interior do estado do Rio Grande do Sul, onde a idade materna média foi de 28,1 anos, com realização de uma média de 7 consultas prénatais durante a gestação, predomínio de neonatos do sexo feminino, com idade gestacional de 34 semanas, sendo a prematuridade associada à Doença da Membrana Hialina a principal indicação de internação na UTIN. O desfecho final dos recém-nascidos internados na UTIN foi alta para enfermaria.

Neste contexto, o estudo visa fornecer subsídios científicos que possam levar a um maior conhecimento, não somente para os profissionais atuantes com estes indivíduos, mas também para seus familiares, permitindo uma assistência especializada no cuidado desta população tão específica e singular, auxiliando no reconhecimento de suas necessidades e carências, possibilitando um enfrentamento do processo de doença, com diminuição das taxas de morbidade e mortalidade, melhora na sobrevida e proporcionando melhor qualidade de vida para estas crianças e suas famílias. 


\section{REFERÊNCIAS}

1. Cruz DCS, Symam NS, Spíndola T. Os cuidados imediatos prestados ao recém-nascido e a promoção do vínculo mãe-bebê. Rev Esc Enferm USP, 2007;41(4):690-7. Disponível em: http://dx.doi.org/10.1590/S0080-62342007000400021

2. Rodrigue VBM, Belham A. Perfil dos recém-nascidos admitidos na uti neonatal do ho spital santo antônio, blumenau/sc, entre 2014-2016. Arq. Catarin Med. 2017 out-dez; 46(4):43-9. Disponível em: http://www.acm.org.br/acm/seer/index.php/arquivos/ article/view/188/203

3. Gama SGN, Szwarcwald CL, Leal MC, Theme Filha MM. Gravidez na adolescência como fator de risco para baixo peso ao nascer no município do Rio de Janeiro, 1996 a 1998. Rev. Saúde Púb., 2001; 35(1):7480. Disponível em: https://doi.org/10.1590/S003489102001000100011

4. Basso CG, Neves ET, Silveira A. Associação entre realização de pré-natal e morbidade neonatal. Texto Contexto Enferm, 2012 Abr-Jun; 21(2): 269-76. Disponível em: https://doi.org/10.1590/S0104$\underline{07072012000200003}$

5. Reis ACF, Cavalcante SKD, Santos ILF. Perfil epidemiológico das infecções hospitalares em uma unidade de terapia intensiva neonatal de um hospital de Cuiabá. [Monografia]; enfermagem, 2018.

6. Marques GM, Pieszak GM, Arrué AM, Rodrigues AP, Gomes GC, Soares RK. Perfil epidemiológico de neonatos de uma unidade de terapia intensiva. REAS, Rev Eletr Acervo Saúde, 2018; 10(6): 2320-8. Disponível em: https://www.acervosaude.com.br/doc/REAS426.pdf

7. Alban ES, Araujo JH, Martins AV, Moraes MV, Maciel V. Cesárea eletiva: Complicações maternas e fetais. ACM arq.catarin. med., 2009; 38(1):45-9. Disponível em: http://www.acm.org.br/revista/index revista.php

8. Ministério da Saúde (BR), Secretária de Atenção a Saúde. Departamento de ações programáticas e estratégicas. Atenção à Saúde de recém-nascido: Guia prático para os profissionais de saúde. Brasília, 2011.
[Acesso em: 20 out. 2016]. Disponível em: http://www.redebhl.fiocruz.br/

9. Freitas PF, Sakae TM, Jacomino MEMLP. Medical and non medical factors associated with cesarean section rates in a university hospital in southern Brazil. Cad Saúde Pública. Rio de Janeiro, 2008; 24(5):1051-61. Disponível em:

https://www.scielosp.org/pdf/csp/2008.v24n5/10511061/pt

10. Ministério da Saúde (BR), Pré-natal e puerpério. Atenção qualificada e humanizada. Brasília: Ministério da Saúde; 2006.

11. Tase TH. Caracterização das mulheres atendidas em um hospital-escola referência para gestação de alto risco. Dissertação (Mestrado em Enfermagem) Universidade de São Paulo/Escolade Enfermagem da USP, São Paulo, 2000.

12. Victora CM, Cesar JA. Saúde materno infantil no Brasil - padrões de morbidade e possíveis intervenções. In: Rouyquariol MZ; Almeida Filho N. Epidemiologia e saúde. 6. ed. Rio de Janeiro: Medsi,p.415-67, 2003.

13. Romero JA, Simão AB, Souza IM. Resultados perinatais de nascidos vivos de mães adolescentes e adultas: uma análise exploratória do município de Belo-Horizonte. Fundação Pinheiros. Rev. Pesq. Saúde, 2010; 11(3):35-40. Disponível em:

http://www.cedeplar.ufmg.br/seminarios/seminario d iamantina/2010/D10A012.pdf

14. Ministério da Saúde (BR), Gestação de alto risco: manual técnico. 4a ed. Brasília (DF): O Ministério; 2000.

15. Ximenes FMA, Oliveira MCR. A influência da idade materna sobre as condições perinatais. Rev.

Brasil.Pesq.Saúde, 2004; 17(2):52-60. Disponível em: https://periodicos.unifor.br/RBPS/article/view/677

16. Delpisheh A, Brabin L, Attia E, Brabin BJ. Pregnancy late in life: a hospital-based study of birth outcomes. J. W. Health, 2008;17(6):956-70. Disponível em: https://dx.doi.org/10.1089\%2Fjwh.2007.0511 
17. Gargus RA, Vohr BR, Tyson JE, High P, Higgins HD, Wrage LA, et al. Unimpaired outcomes for extremely low birth weight infants at 18 to 22 months. Pediatrics, 2009; 124(1):112-21. Disponível em:

https://doi.org/10.1542/peds.2008-2742

18. Peralta-Carcelen M, Moses M, Adams-Chapman I, Gantz M, Vohr BR. Stability of neuromotor outcomes at 18 and 30 months of age after extremely low birth weight status. Pediatrics, 2009; 123(5):887-95. Disponível em: https://doi.org/10.1542/peds.2008$\underline{0135}$

19. Loth E, Vitti C, Nunes Jl. A diferença das notas do escore de Apgar entre crianças nascidas de parto normal e parto cesariana. Arq. Ciên. Saúde (Unipar), 2001; 5(3):211-13. Disponível em: https://www.revistas.unipar.br/index.php/saude/articl e/view/1131

20. Santoro W, Martinez FE. Precisão do escore de Apgar no atendimento ao recém-nascido de muito baixo peso. Rev. Paulist. Pediat., 2004; 22(4):193-7. Disponível em:

21. Cunha AL, Fernandes DS, Melo PF, Guedes MH. Fatores associados à asfixia perinatal. Ver. Bras.Ginec.Obst., 2004;26(10):799-805. Disponível em: https://pesquisa.bvsalud.org/portal/resource/en/lil412836

22. Muniz DWR, Miranda MG, Lima GWF, Costa A P, Vale EA. Perfil epidemiológico dos óbitos neonatais da unidade de terapia intensiva. Rev enferm UFPE on line. 2018; 12(9):2393-8. Disponível em:

https://periodicos.ufpe.br/revistas/revistaenfermagem Larticle/view/230758/29928

23. Tsushima K, King LS, Aggarwal NR, De Gorordo A, D'alessio FR, Kubo K. Acute lung injury review. Intern Med., 2009; 48(9):621-30. Disponível em: https://doi.org/10.2169/internalmedicine.48.1741

24. Sociedade Brasileira de Pediatria. Consenso Brasileiro em Ventilação Mecânica: Suporte ventilatório na síndrome do desconforto respiratório do recém-nascido. [Acesso em: 26 out. 2016]. Disponível em: http://www.sbp.com. br/pdfs/sdr.pdf.

25. Nardelli LM, Garcia C, Pássaro CP, Rocco PR. Entendendo os mecanismos determinantes da lesão pulmonar induzida pela ventilação mecânica. Rev. Bras. Ter. Inten. 2007; 19(4):469-74. Disponível em: http://dx.doi.org/10.1590/S0103-507X2007000400011

26. Vrijlandt EJ, Gerritsen J, Boezen HM, Duiverman EJ, Dutch Pops. Collaborative Study Group. Gender differences in respiratory symptoms in 19-year-old adults born preterm. Respir Res,2005; 6(1):117. Disponível em: https://doi.org/10.1186/1465-9921-6$\underline{117}$

27. Araujo LZS, Neves Júnior WA. A bioética e a fisioterapia nas Unidades de Terapia Intensiva. Rev. Fisioter. Univ. São Paulo, 2003; 10(2):52-60. Disponível em:

http://www.revistas.usp.br/fpusp/article/view/78115

28. All-Alaiyan S, Dyer D and Khan B. Chest physiotherapy and pos-extubation atelectasis in infants. Pediatric Pulmonology.1996; 21(4):227-30. Disponível em: https://doi.org/10.1002/(sici)10990496(199604)21:4\%3C227::aid-ppul4\%3E3.0.co;2-I

29. Martins AP, Segre CAM. Fisioterapia Respiratória em neonatologia: importância e cuidados. Pediat. Mod., 2010.

30. Tiago LF, Caldeira AP, Vieira MA. Fatores de risco de baixo peso ao nascimento em maternidade pública do interior de Minas Gerais. Pediatria, São Paulo, 2008;30(1):8-14. Disponível em:

Sources of funding: No

Conflict of interest: No

Accepted: 2019/02/06

Publishing: 2019/12/08

Corresponding Address: Débora D'Agostini Jorge Lisboa

Endereço: Rua Tiradentes 295, CEP: 99010-260, centro, Passo Fundo, RS.

Telefone: (54) 98126-4877

E-mail: debydi@yahoo.com.br 